\title{
Evidence of high-risk sexual behaviors among injection drug users in the Kenya PLACE Study
}

\author{
Paul Brodisha, ${ }^{a}$, Kavita Singha, ${ }^{a, b}$, Agnes Rinyurid $^{d}$, Carol Njerud ${ }^{d}$, Nzioki Kingolad ${ }^{d}$, Patrick \\ Mureithie, William Sambisa ${ }^{\dagger}$, and Sharon Weir ${ }^{a, c}$ \\ aMEASURE Evaluation, Carolina Population Center, The University of North Carolina at Chapel \\ Hill, 123 W. Franklin Street, Chapel Hill, NC 27516-2524, USA \\ bDepartment of Maternal and Child Health, Gillings School of Global Public Health, The University \\ of North Carolina at Chapel Hill, 135 Dauer Drive, Chapel Hill, NC 27599-7400, USA \\ 'Department of Epidemiology, Gillings School of Global Public Health, The University of North \\ Carolina at Chapel Hill, 135 Dauer Drive, Chapel Hill, NC 27599-7400, USA \\ dInternational Center for Reproductive Health, P.O. Box 91109 - 80103, Mombassa, Kenya \\ eNational AIDS Control Commission, Landmark Plaza, $9^{\text {th }}$ Floor, Argwings Kodhek Road, P.O. \\ Box 61307 - 00200, Nairobi, Kenya \\ fAIDSTAR-TWO, Management Sciences for Health, 4301 North Fairfax Drive, Arlington, VA \\ 22203, USA
}

\section{Abstract}

Background-Injection drug users (IDUs) in resource poor settings are at high risk for HIV transmission through unsafe needle-sharing and sexual practices. We report on the injecting and sexual behavior of a sample of IDUs from Malindi, Kenya.

Methods-A Priority for Local AIDS Control Efforts (PLACE) study was conducted from April to May 2010 to identify areas where HIV transmission is most likely to occur and specific venues where people meet new sexual partners. Community informants $(n=202)$ listed 157 unique venues from which 29 were randomly selected using a systematic fixed interval sampling strategy with probability of selection proportional to venue size. Twenty patrons and four workers were interviewed at each venue. Drug use practices were elicited in a staff-administered interview.

Results-Between $40 \%$ and 50\% of IDUs reported needle-sharing, taking drugs from a common reservoir, using a ready-made solution without boiling, and not exchanging a used for a new syringe in the past month. Most could inconsistently or never get new syringes. In multivariate logistic regression models controlling for age, education, residence, and poverty status, IDUs were twice as likely as non-IDUs to report multiple partners in the past year (OR 1.94, 95\% CI $1.26-$ $3.00, \mathrm{p}<.01$ ) and multiple new partners in the past year (OR 2.11, 95\% CI $1.30-3.42, \mathrm{p}<.01$ ).

Conclusions-High prevalence of multiple sexual partnerships and risky injecting behaviors among IDUs and unavailability of new injecting needles are likely facilitating HIV transmission in Malindi, Kenya.

\footnotetext{
(C) 2011 Elsevier Ireland Ltd. All rights reserved.

*Corresponding author: Telephone: (919) 966-3510; Fax: (919) 966-2391 brodish@unc.edu.
}

Publisher's Disclaimer: This is a PDF file of an unedited manuscript that has been accepted for publication. As a service to our customers we are providing this early version of the manuscript. The manuscript will undergo copyediting, typesetting, and review of the resulting proof before it is published in its final citable form. Please note that during the production process errors may be discovered which could affect the content, and all legal disclaimers that apply to the journal pertain. 


\section{Key words or phrases}

injection drug use; HIV-1; sub-Saharan Africa; sexual behavior

\section{Introduction}

Although the published epidemiological literature is limited, evidence suggests injection drug use is becoming increasingly common in sub-Saharan Africa (SSA) (United Nations Office on Drugs and Crime, 2010a). It is already well-established in Kenya, Mauritius, South Africa, Nigeria, and Tanzania, with estimated prevalence rates between $0.5 \%$ and $1 \%$ in Kenya and South Africa and exceeding $2 \%$ on the island of Mauritius, though remarkably, only three of 47 SSA countries have reliable national prevalence data (Mathers et al., 2008; United Nations Office on Drugs and Crime, 2010a). Several factors presage its more widespread development in the region, including an increase in transit of illicit drugs (heroin from Asia and cocaine from South America) into Europe through SSA countries with unmonitored and porous borders (United Nations Office on Drugs and Crime, 2010b), and endemic cofactors such as socioeconomic hardship, political instability, and exposure to conflicts.

Limited data on IDU behaviors in SSA indicates sharing injecting equipment is common; access to clean water for cleaning equipment and mixing injecting solutions is limited; and many do not self-identify as injection drug users (IDUs), some repeatedly switching between injecting and non-injecting modes (United Nations Office on Drugs and Crime, 2010a). And although even fewer studies report on prevalence of HIV among IDUs (Mathers et al., 2008), SSA is home to two-thirds of people living with HIV/AIDS or 22.5 million people (Joint United Nations Programme on HIV/AIDS, 2010), with prevalence rates well above $2 \%$ in 14 of the 16 countries where injecting drug use has been reported (United Nations Office on Drugs and Crime, 2010a). Given the high regional HIV prevalence and the efficiency of HIV transmission per injection - six times higher than for heterosexual acts (United Nations Office on Drugs and Crime, 2005) - HIV prevalence among IDUs can rapidly reach very high levels (more than 50\% and up to 90\%) (United Nations Office on Drugs and Crime, 2010a). Research in African countries has found HIV prevalence among IDUs far exceeding that in the general population, ranging from $9 \%$ to $50 \%$ (Needle et al., 2006). Further, IDUs have been identified as a bridging population (Beckerleg and Hundt, 2004; Stimson and Choopanya, 1998) with an important role in spreading HIV to the general population through transmission to sexual partners and vertically from mother to child.

In Kenya, IDU has been confirmed in Nairobi, Mombasa, Malindi, and Lamu (the latter three in Coast province). HIV prevalence among heroin users was reported to be $35 \%$ (Odek-Ogunde et al., 2004), and another study indicated a prevalence approximately five times that of the general population (Deveau et al., 2006). According to a modes of transmission (MoT) study in Kenya, the percent of new infections attributable to IDU is $3.8 \%$ nationally, 5.8\% in Nairobi, and 6.1\% in Coast Province (National AIDS Control Council, 2009). Although the percent of incident cases attributable to IDU is relatively low, modeling indicates that the incidence rates in the Kenyan HIV epidemic are highest among IDU at 256 per 1,000 (or 25.6\%), followed by MSM in prison (126/1,000), partners of IDU $(78 / 1,000)$, and MSM (67/1000). Besides the highly efficient transmission through needlesharing and other risky practices, the high incidence rate in IDUs reflects marginalization and inaccessibility of targeted interventions in this group (National AIDS Control Council, 2009). 
Several studies indicate that IDUs are at high risk for HIV transmission through unsafe practices such as sharing non-sterile injecting equipment and unprotected sex (McCurdy et al., 2005; Ndetei, 2004; Odek-Ogunde et al., 2004; Parry et al., 2009). Prior research on IDUs in countries with similar profiles (e.g., Tanzania, Mozambique, and South Africa) has typically focused on distinct high risk sub-populations, has often been qualitative, and where quantitative-because of sample size constraints or the nature of the sub-populations included - has rarely allowed for a strong comparison of sexual risk behaviors between injection and non-injection drug users. To the extent that IDUs engage in more high-risk sexual behavior with multiple, concurrent partners, compared to non-IDUs, they potentiate the bridging of HIV from IDU to non-IDU populations and extend or prolong the generalized epidemic. This brief report characterizes the sexual behavior of a sample of IDUs in the coastal town of Malindi, Kenya and compares sexual risk behaviors among a sample of IDUs and non-IDUs in order to add to our limited knowledge of this important and growing source of new HIV cases in Kenya.

\section{Methods}

A Priority for Local AIDS Control Efforts (PLACE) study was conducted in Malindi, Kenya from April to May 2010. The PLACE methodology is focused on identifying the areas where HIV transmission is most likely to occur - priority prevention areas (PPAs) - and identifying specific venues within the PPAs where people meet new sexual partners and where IDUs can be reached. This methodology is based on epidemiology theory which indicates that there is often geographic clustering of HIV transmission (Grassly et al., 2001; Wasserheit and Aral, 1996; Weir et al., 2005) and has been described elsewhere (Weir et al., 2004). The PLACE method has been implemented in over 60 countries and has been shown to be more effective in capturing the individuals with riskier sexual behaviors compared to household surveys (Speizer et al., 2009; Tate et al., 2010; Weir et al., 2004).

Briefly, PLACE involves identifying venues and events where people are believed to frequent to meet new sexual partners and places where IDUs can be reached. Interviewers approach potential community informants (types of people, based on occupation, identified by the local PLACE implementing team as being knowledgeable about venues in the PPA) and request that they list the places where people are believed to meet new sexual partners and where IDUs can be reached in their local area. Informants were also asked where IDUs specifically meet new partners. Common types of informants were health care workers, beer/liquor store owners, mobile hawkers/street vendors, taxi drivers, program coordinators, peer educators, and business people.

Each venue/event was visited and verified: 202 community informants listed 157 unique venues. At each of the venues a representative was surveyed to obtain information about the venue, the people who socialize there, existing HIV prevention activities, and the willingness to have such activities. The venue representative was typically an owner or manager or someone who worked at the venue (or near the venue in the case of open spaces) and was knowledgeable about the people who typically socialize at that venue. The venue representative reported the venue size, which was considered to be the total number of persons socializing at the venue during a busy time.

Next, the characteristics of people socializing or working at the sites were obtained via survey sampling. Venues/events were randomly selected using a systematic fixed interval sampling strategy with the probability of selection proportional to the size of the venue. Venues and events were listed by both geographic code and size. Large venues could be selected more than once if their cluster code number (determined by venue size) was larger than the selection interval. The systematic fixed interval sampling strategy produced a self- 
weighted sample which gives all individuals socializing and working at eligible venues an equal probability of selection for an individual interview. Probability proportional-to-size was then used to select 29 venues/events in Malindi. An additional 10 venues were identified by community informants as places where IDUs socialize. All of these venues were visited to ensure a sufficient sample size of IDUs.

Local interviewers were trained on the rationale, objectives and methods for systematically selecting and interviewing venue patrons, and they were provided a step-by-step interviewer guide explaining how to complete each interview question. Interviewers selected 20 individuals (10-13 men, 7-10 women) to interview at each of the selected venues and 4 workers ( 2 male, 2 female). The numbers of men and women to interview were based on the gender composition of socializers at each venue, which was obtained from the venue representative survey. For those sites with more than 20 individuals, interviewers systematically selected every $\mathrm{i}^{\text {th }}$ individual socializing along an imaginary diagonal line traversing different locations within the venue, where $\mathrm{i}$ is the sampling interval, or total number of individuals at the venue divided by the number of interviews (20) required. For those sites with fewer than 20 individuals and fewer than four workers, all were interviewed. At some of the very small venues, interviewers waited for more socializers to arrive. All individuals aged 18 and older were eligible for interview. Those aged 16 or 17 could be interviewed if they were at the venue as a socializer without a parent (acting as an adult) and not on a family errand. IDUs were asked to self-identify and were restricted to those indicating they had injected drugs within the past year. Injecting practices of IDUs were obtained via an Injection Drug Use Module including five (in some cases multipart) questions eliciting drug injecting behaviors. The module included questions about how the respondent typically injects (individually, with the same group, with different groups); whether he/she shared a syringe at last injection; whether he/she shared a syringe, injected from a common reservoir, used a ready-made solution without boiling, or exchanged a used for a new syringe in the past month; the total number of people with whom he/she shared a syringe in the past month, and of those, the number with whom he/she shared for the first time; and whether new syringes are available when wanted. The module did not differentiate between receptive needle sharing (risk to self) and distributive needle sharing (risk to others), an acknowledged limitation of our study. Ethical approval was given by the Kenyatta National Hospital Ethical Review Committee and the University of North Carolina at Chapel Hill Institutional Review Board.

The study included voluntary testing and counseling (VCT) for HIV. Study participants were told at the beginning and again at the end of the survey that they could be tested for $\mathrm{HIV}$ if they chose to do so. Testing was done immediately following the survey in most cases. Those who agreed to be tested were taken to a quiet room in the venue or a nearby venue. In some cases, a tent was pitched outside the venue for privacy. VCT was done by experienced and trained counselors. Individuals could receive their results immediately or could call for their results at a later time. All HIV positive individuals were referred to the nearest VCT center for follow-up counseling and guidance on treatment options. Some individuals chose to answer the questionnaire but were not reached for HIV testing. Information on HIV prevalence among IDUs was not available at the time of writing.

The analysis sample was restricted to non-MSM males because the IDUs were almost exclusively male and because we were interested in isolating the risk in IDU versus nonIDU populations. We performed simple univariate analyses of the IDU Module variables in the IDU population and bivariate comparisons on IDU status for the demographic and sexual risk behavior variables. A composite indicator meant to capture sexual risk is often presented in PLACE studies. This indicator is based on the number of new and total partners reported in the past 4 weeks and 12 months. Respondents are classified as having high, 
moderate or low rates of sexual partnerships based on the following classification: high new partner in past 4 weeks or multiple partners in past 4 weeks; moderate - new or multiple partners in the past 12 months; low - no new partners or no multiple partners in past 12 months. Rank sum and chi-square tests are used to test for statistical significance in bivariate analyses. Multivariate logistic regressions were performed using number of sexual partners and new sexual partners in the past year as dependent variables, IDU status as the predictor variable, and age, secondary or greater education level, urban/rural residence, and poverty status as covariates. In keeping with international definitions, a person was considered to be living in poverty if he/she lived on less than $\$ 1.25$ per day. Poverty was self-reported using the survey question "How much money did you make from all sources in the past 4 weeks? (Include income from all sources)" and reported in Kenya Shillings.

\section{Results}

Demographic and sexual risk behavior characteristics of the non-MSM male IDUs and nonIDUs are shown in Table 1. IDUs in Malindi were more likely to be 34+ years old $(\mathrm{p}<.01)$, less educated $(\mathrm{p}<.001)$, and urban residents $(\mathrm{p}<.01)$. They were also more likely to report two or more sexual partners in the past year $(\mathrm{p}<.05)$, two or more new partners in the past year $(\mathrm{p}<.01)$, and a higher composite sexual risk score $(\mathrm{p}<.05)$ compared to non-IDUs.

Over $70 \%$ of IDUs reported injecting within the past week and $84 \%$ reported injecting within the past month (Table 2). The majority (52\%) reported injecting alone and another $33 \%$ reported usually injecting with the same group. Nearly half (48\%) indicated that they shared a syringe the last time they injected, and almost the same percentage reported sharing a syringe in the past month. During the past month $43 \%$ reported taking drugs from a common reservoir, $49 \%$ used a ready-made solution without boiling, and $44 \%$ had not exchanged a used for a new syringe. Over $20 \%$ of the IDUs reported sharing a syringe with between one and three different people in the past month, $6 \%$ reported sharing with four to five different people, $8 \%$ reported sharing with six to nine different people, and $6 \%$ reported sharing with 10 or more different people. Of those who reported sharing a syringe, $23 \%$ reported sharing with one to three different people for the first time, and another $4 \%$ with between four and 10 different people for the first time. Most IDUs reported that they could only inconsistently or never get new syringes when they wanted them. In multivariate logistic regression models controlling for age, secondary education or higher, urban/rural residence, and poverty status, IDUs were twice as likely as non-IDUs to report multiple partners in the past year (OR $1.94,95 \%$ CI $1.26-3.00, \mathrm{p}<.01$ ) and multiple new partners in the past year (OR $2.11,95 \%$ CI $1.30-3.42, \mathrm{p}<.01)$.

\section{Discussion}

This brief report confirms the increased prevalence of multiple sexual partnerships and new partnerships, the high prevalence of risky drug injecting behaviors, and the difficulty obtaining new injecting needles in a sample of IDUs in Malindi, Kenya, all of which increase the likelihood of HIV transmission. It is unclear exactly why IDUs were twice as likely to engage in risky sexual practices. A qualitative study in South Africa (Parry et al., 2009) detected a complex, context-specific relationship between drug use and sexual behaviors and provides some insights. Many IDUs in that study used heroin, a depressant that tends to inhibit libido, especially in large quantities or with long-term use, but additionally consumed stimulant-type drugs such as crystal methamphetamine and Ecstasy that increased sexual desire. Sexually active interviewees reported more sexual partners (which included regular partners, friends, drug dealers, and strangers) when on drugs. Males (both non-IDUs and IDUs) exchanged drugs for sex with sex workers for free or with sexually available women, even if they were already involved in relationships. And despite a 
general awareness that unsafe sex could lead to HIV, substance use impaired judgment regarding condom use and other safe sexual practices. A recent study among IDUs in Tanzania reported an HIV seroprevalence rate of $42 \%$, and in multivariate models the strongest independent predictor of testing HIV-positive was having had sex 81 or more times in the past month, which increased the probability approximately six times (Williams et al., 2009).

Such findings point to an urgent need to seek out this marginalized population with the nine proven effective interventions for the prevention, treatment, and care of HIV in IDUs endorsed by UNAIDS, WHO, and UNODC (WHO et al., 2009): needle and syringe programmes; opioid substitution therapy and other drug dependence treatment; HIV testing and counselling; antiretroviral therapy; prevention and treatment of sexually transmitted infections; condom programmes for IDUs and their sexual partners; targeted information, education and communication for IDUs and their sexual partners; vaccination, diagnosis and treatment of viral hepatitis; and prevention, diagnosis and treatment of tuberculosis. A welcomed recent development in this direction is that the Kenyan Government has agreed to provide such a comprehensive package of HIV services for IDUs, beginning in Mombasa and extending to other regions of the country (United Nations Office on Drugs and Crime, 2011). A recent modeling study indicates that eliminating laws prohibiting opioid substitution and scaling up of such services to $80 \%$ coverage in Nairobi could prevent $14 \%$ of incident HIV infections in IDUs (Strathdee et al., 2010).

\section{References}

Beckerleg SE, Hundt GL. The characteristics and recent growth of heroin injecting in a Kenyan coastal town. Addict Res Theory. 2004; 12:41-53.

Deveau C, Levine B, Beckerleg S. Heroin use in Kenya and findings from a community based outreach programme to reduce the spread of HIV/AIDS. African J Drug Alcohol Stud. 2006; 5:95107.

Grassly NC, Garnett GP, Schwartlander B, Gregson S, Anderson RM. The effectiveness of HIV prevention and the epidemiological context. Bull WHO. 2001; 79:1121-1132. [PubMed: 11799444]

Joint United Nations Programme on HIV/AIDS. Global Report: UNAIDS Report on the Global AIDS Epidemic 2010. Geneva: 2010.

Mathers BM, Degenhardt L, Phillips B, Wiessing L, Hickman M, Strathdee SA, Wodak A, Panda S, Tyndall M, Toufik A, Mattick RP. Reference Grp, U.N.H.I.V.I. Global epidemiology of injecting drug use and HIV among people who inject drugs: a systematic review. Lancet. 2008; 372:17331745. [PubMed: 18817968]

McCurdy SA, Williams ML, Kilonzo GP, Ross MW, Leshabari MT. Heroin and HIV risk in Dar es Salaam, Tanzania: youth hangouts, mageto and injecting practices. Aids Care Psychol Socio-Med Asp AIDS-HIV. 2005; 17:S65-S76.

National AIDS Control Council. Kenya HIV Prevention Response and Modes of Transmission Analysis: Final Report. Nairobi, Kenya: 2009.

Ndetei, D. Study on the Assessment of the Linkages Between Drug Abuse, Injecting DrugAbuse and HIV/AIDS in Kenya: A Rapid Situation Assessment 2004. United Nations Office on Drugs and Crime; Nairobi, Kenya: 2004.

Needle RH, Kroeger K, Belani H, Hegle J. Substance abuse and HIV in Sub-Saharan Africa: introduction to the special issue. African J Drug Alcohol Stud. 2006; 5:83-95.

Odek-Ogunde, M.; Okoth, FA.; Lore, W.; Owiti, FR. Seroprevalence of HIV, HBC and HCV in injecting Drug Users in Nairobi, Kenya: World Health Organization Drug Injecting Study Phase II Findings. 15th International AIDS Conference; Bangkok. 2004.

Parry CDH, Carney T, Petersen P, Dewing S, Needle R. HIV-risk behavior among injecting or nonInjecting drug users in Cape Town, Pretoria, and Durban, South Africa. Subst Use Misuse. 2009; 44:886-904. [PubMed: 19444728]

Drug Alcohol Depend. Author manuscript; available in PMC 2012 December 1. 
Speizer IS, Beauvais H, Gomez AM, Outlaw TF, Roussel B. Using multiple sampling approaches to measure sexual risk-taking among young people in Haiti: programmatic implications. Stud Fam Plan. 2009; 40:277-288.

Stimson, G.; Choopanya, K. Global perspectives on drug injecting. In: Stimson, G.; Des Jarlais, DC.; Ball, A., editors. Drug Injecting and HIV Infection. UCL Press; London: 1998.

Strathdee SA, Hallett TB, Bobrova N, Rhodes T, Booth R, Abdool R, Hankins CA. HIV and risk environment for injecting drug users: the past, present, and future. Lancet. 2010; 376:268-284. [PubMed: 20650523]

Tate J, Singh K, Ndubani P, Kamwanga J, Buckner B. Measurement of HIV prevention indicators: a comparison of the PLACE method and a household survey in Zambia. AIDS Behav. 2010; 14:209-217. [PubMed: 19089607]

United Nations Office on Drugs and Crime. 2005 World Drug Report. Vienna: 2005.

United Nations Office on Drugs and Crime. Consensus Statement of the Reference Group to the United Nations on HIV and Injecting Drug Use. Vienna: 2010a.

United Nations Office on Drugs and Crime. World Drug Report 2010. Vienna: 2010b.

United Nations Office on Drugs and Crime. [accessed on March 21, 2011] Kenya Government to provide HIV services for people who inject drugs. 2011. http://www.unodc.org/unodc/en/hiv-aids/about-kenya.html

Wasserheit JN, Aral SO. The dynamic topology of sexually transmitted disease epidemics: implications for prevention strategies. J Infect Dis. 1996; 174:S201-S213. [PubMed: 8843250]

Weir, S.; Tate, J.; Bassett Hileman, S.; Khan, M.; Jackson, E.; Johnston, A.; Herman, C. MEASURE Evaluation. US-AID; Washington: 2005. A Manual for Implementing the PLACE Method.

Weir SS, Tate JE, Zhusupov B, Boerma JT. Where the action is: monitoring local trends in sexual behaviour. Sex Transm Infect. 2004; 80:63-68. [PubMed: 14755040]

WHO, UNODC, UNAIDS. Technical Guide for Countries to set Targets for Universal Access to HIV Prevention, Treatment and Care for Injecting Drug Users. World Health Organization; Geneva: 2009.

Williams ML, McCurdy SA, Bowen AM, Kilonzo GP, Atkinson JS, Ross MW, Leshabari MT. HIV seroprevalence in a sample of Tanzanian intravenous drug users. AIDS Educ Prev. 2009; 21:474483. [PubMed: 19842830] 


\section{Table 1}

Demographic and sexual risk behavior characteristics of non-MSM male IDUs vs. non-IDUs in the Malindi PLACE sample

\begin{tabular}{|c|c|c|}
\hline & Non-IDUs N=377 & IDUs $\mathrm{N}=124$ \\
\hline & $n(\%)$ & $n(\%)$ \\
\hline \multicolumn{3}{|l|}{ Age } \\
\hline $15-24$ & $120(31.9)$ & $21(16.9)$ \\
\hline $25-34$ & $169(45.0)$ & $69(55.6)$ \\
\hline $34+$ & $87(23.1)$ & $34(27.4)^{* *}$ \\
\hline \multicolumn{3}{|l|}{ Currently working } \\
\hline Yes & $335(89.1)$ & $111(90.2)$ \\
\hline No & $41(10.9)$ & $12(9.8)$ \\
\hline \multicolumn{3}{|c|}{ Highest level of education } \\
\hline None & $68(18.0)$ & $47(38.2)$ \\
\hline Primary & $157(41.6)$ & $57(46.3)$ \\
\hline Secondary+ & $152(40.3)$ & $19(15.4)^{* * *}$ \\
\hline \multicolumn{3}{|l|}{ Residence } \\
\hline Urban & $345(92.7)$ & $122(99.2)$ \\
\hline Rural & $27(7.3)$ & $1(0.8)^{* *}$ \\
\hline \multicolumn{3}{|l|}{ Marital status } \\
\hline Ever married & $212(57.4)$ & $73(59.4)$ \\
\hline Never married & $157(42.6)$ & $50(40.6)$ \\
\hline \multicolumn{3}{|l|}{ Poverty status } \\
\hline In poverty & $56(15.0)$ & $17(13.8)$ \\
\hline Not in poverty & $316(85.0)$ & $106(86.2)$ \\
\hline \multicolumn{3}{|c|}{ Number of partners in past 4 weeks } \\
\hline 0 & $103(27.4)$ & $38(30.6)$ \\
\hline 1 & $200(53.2)$ & $49(39.5)$ \\
\hline $2+$ & $73(19.4)$ & $37(29.8)$ \\
\hline \multicolumn{3}{|c|}{ Number of partners in past 12 months } \\
\hline 0 & $31(8.2)$ & $13(10.5)$ \\
\hline 1 & $187(49.7)$ & $40(32.3)$ \\
\hline $2+$ & $158(42.0)$ & $71(57.3)^{*}$ \\
\hline \multicolumn{3}{|c|}{ Number of new partners in past 4 weeks } \\
\hline 0 & $283(75.7)$ & $91(73.4)$ \\
\hline 1 & $70(18.7)$ & $26(21.0)$ \\
\hline $2+$ & $21(5.6)$ & $7(5.6)$ \\
\hline \multicolumn{3}{|c|}{ Number of new partners past 12 months } \\
\hline 0 & $207(55.4)$ & $50(40.3)$ \\
\hline
\end{tabular}




\begin{tabular}{|l|r|r|}
\hline & Non-IDUs N=377 & IDUs N=124 \\
\hline 1 & $\boldsymbol{n}(\boldsymbol{\%})$ & $\boldsymbol{n}(\boldsymbol{\%})$ \\
\hline $2+$ & $90(24.1)$ & $34(27.4)$ \\
\hline Given/received money for sex in past 12 months & $77(20.6)$ & $40(32.3)^{* *}$ \\
\hline No & $300(81.1)$ & $100(82.6)$ \\
\hline Yes & $70(18.9)$ & $21(17.4)$ \\
\hline Condom use at last intercourse & & \\
\hline No & $291(80.8)$ & $107(87.7)$ \\
\hline Yes & $69(19.2)$ & $15(12.3)$ \\
\hline Composite risk & & \\
\hline Low & $176(46.7)$ & $44(35.5)$ \\
\hline Moderate & $90(23.9)$ & $32(25.8)$ \\
\hline High & $111(29.4)$ & $48(38.7)^{*}$ \\
\hline
\end{tabular}

${ }^{*} P<0.05$,

${ }^{* *} P<0.01$,

**** $P<0.001$ 
Table 2

Characteristics of non-MSM male IDUs in the Malindi PLACE sample

\begin{tabular}{|c|c|}
\hline & $\mathrm{n}(\%)$ \\
\hline \multicolumn{2}{|l|}{ When last injected drugs } \\
\hline Within past 7 days & $91(73.4)$ \\
\hline Within past $2-4$ weeks & $13(10.5)$ \\
\hline Within past 2-6 months & $12(9.7)$ \\
\hline Within past $7-12$ months & $8(6.4)$ \\
\hline \multicolumn{2}{|c|}{ With whom do you usually inject drugs? } \\
\hline Individually & $64(52.5)$ \\
\hline Usually with the same group & $40(32.8)$ \\
\hline With different groups & $15(12.3)$ \\
\hline Depends on circumstances & $3(2.5)$ \\
\hline \multicolumn{2}{|l|}{ Shared syringe last time injected } \\
\hline Yes & $58(47.5)$ \\
\hline No & $64(52.5)$ \\
\hline \multicolumn{2}{|l|}{ During the past 4 weeks... } \\
\hline \multicolumn{2}{|l|}{ Shared a syringe } \\
\hline Yes & $50(42.0)$ \\
\hline No & $69(58.0)$ \\
\hline \multicolumn{2}{|c|}{ Took drugs from common reservoir } \\
\hline Yes & $51(42.9)$ \\
\hline No & $68(57.1)$ \\
\hline \multicolumn{2}{|c|}{ Used ready-made solution without boiling } \\
\hline Yes & $58(48.7)$ \\
\hline No & $61(51.3)$ \\
\hline \multicolumn{2}{|c|}{ Exchanged a used for a new syringe } \\
\hline Yes & $66(55.9)$ \\
\hline No & $52(44.1)$ \\
\hline \multicolumn{2}{|c|}{ Number of different people you shared a syringe with } \\
\hline 0 & $69(56.1)$ \\
\hline $1-3$ & $29(23.6)$ \\
\hline $4-5$ & $8(6.5)$ \\
\hline $6-9$ & $10(8.1)$ \\
\hline $10+$ & $7(5.7)$ \\
\hline \multicolumn{2}{|c|}{ Number of those people you shared a syringe with for the first time } \\
\hline 0 & $90(73.2)$ \\
\hline $1-3$ & $28(22.8)$ \\
\hline $4-5$ & $3(2.4)$ \\
\hline $6-9$ & $1(0.8)$ \\
\hline
\end{tabular}




\begin{tabular}{|l|r|}
\hline & $\mathbf{n}(\boldsymbol{\%})$ \\
\hline $10+$ & $1(0.8)$ \\
\hline Can you get new syringes whenever you want? & \\
\hline Always & $55(45.4)$ \\
\hline Sometimes & $62(51.2)$ \\
\hline Never & $4(3.3)$ \\
\hline
\end{tabular}

